\title{
New Dimensionless Numbers Governing Cavitation in Diesel Multi-Hole Mini-Sac Nozzles and Sprays
}

\author{
Samsu Dlukha Nurcholik ${ }^{1}$, Takashi Miwa ${ }^{1}$, Masashi Wakisaka ${ }^{1}$, Kazushi Kimura $^{1}$, \\ Akira Sou*1, \\ Keiya Nishida², Mikimasa Kawaguchi², Yuhei Matsumoto², Yoshitaka Wada ${ }^{3}$ \\ ${ }^{1}$ Graduate School of Maritime Sciences, Kobe University, Kobe, Japan \\ ${ }^{2}$ Graduate School of Engineering, Hiroshima University, Hiroshima, Japan \\ ${ }^{3}$ Powertrain Engineering Analysis Group, Mazda Motor Corporation, Hiroshima, Japan \\ *Corresponding author email: sou@maritime.kobe-u.ac.jp
}

\begin{abstract}
Cavitation along orifice walls, string cavitation in a sac and orifices, and turbulent flows in multihole mini-sac diesel fuel injectors play an essential role in the characteristics of the fuel sprays, which affect the thermal efficiency and exhaust gas emissions of diesel engines. Thus, numerous visualization experiments and numerical simulations on the turbulent cavitation flow in mini-sac nozzles with various geometries and sizes have been carried out, which reported different characteristics of wall cavitation, string cavitation, and sprays. However, we cannot predict quantitatively the turbulent cavitating flows and the sprays since their governing dimensionless indices have not been clarified yet. In this study, we carry out high-speed visualizations of cavitation and liquid jets, high-speed stereo PIV, high-speed PIV, and numerical simulations of turbulent cavitating flows in various transparent multi-hole mini-sac nozzles with different injector sizes, needle lifts, liquid flow rates, nozzle hole numbers, hole diameters, hole positions, needle tip geometries, and liquid fuel properties to clarify the cavitation and flow structure in them as well as spray characteristics. Then, we propose new dimensionless indices, by which we can quantitatively predict the circulation in the sac, string cavitation diameter, cavitation length in an orifice, and the resulting spray angle.
\end{abstract}

\section{Keywords}

Fuel Injector, String Cavitation, PIV, Numerical Simulation, Dimensionless Analysis

\section{Introduction}

It is well known that the cavitation in the nozzle hole of fuel injectors influences the characteristics of the discharged liquid jet and spray, which was first addressed by Bergwerk [1] and plays a vital role in the combustion process and pollutant formation of diesel engines [2]. A lot of visualization experiments on cavitation in transparent injectors clarified that super cavitation in an orifice induced a large and strong turbulence, which increases liquid jet angle and promotes liquid jet atomization [3]-[6]. Recent visualization experiments pointed out that string cavitation in the sac and an orifice of diesel fuel injectors give a larger impact on the angle of injected hollow-cone sprays [7]-[13]. They reported that string cavitation appears intermittently at the moment of low needle lift [9][11][12]. He et al. [9] and Nakamichi et al. [13] observed a steady string cavitation with a large diameter, which increases largely the cone angle of the hollow-cone sprays. Some of the present authors [10][11][12] have manufactured a number of large-scaled transparent multi-hole mini-sac injectors and measured the 2D and $3 \mathrm{D}$ flows in the sacs to clarify the formation of the toroidal vortex flow in the sacs at the moment with string cavitation. In the previous studies, the researchers used various transparent injectors with different injector geometries, injector sizes, needle lifts, needle tip geometries, nozzle hole diameters, hole positions, hole numbers, liquid flow rates, and injection pressures. 
However, since the dimensionless indices which govern the turbulent cavitating flows in the complicated multi-hole mini-sac injectors are not known, we cannot predict quantitatively the flow and spray characteristics, such as the circulation in the sac, string cavitation diameter, cavitation length in a nozzle hole, and the resulting spray angle.

In this study, we carry out a high-speed visualization of cavitation in various injectors and sprays, a high-speed stereo PIV, a high-speed PIV, and a numerical simulation of the turbulent cavitation flows in the multi-hole transparent mini-sac nozzles in order to clarify the effect of needle lifts, liquid flow rates, nozzle hole numbers, hole diameters, hole positions, needle tip geometries, and liquid fuel properties on the cavitation flow and spray. Then, we propose new dimensionless indices, by which we can quantitatively predict the circulation in the sac, string cavitation diameter, cavitation length in an orifice, and the resulting spray angle.

\section{Experimental setup and numerical method}

2.1 Experimental setup

We used the experimental setup shown in Figure 1, which has been used in our previous studies [4][5][10][11][13]. The injection system consists of a pressure vessel, valves, a flow meter, pressure gauges, and a transparent acrylic injector. The needle valve was fixed, and the liquid was injected at constant injection pressure and constant flow rate. Tap water or diesel fuel mixed with about $21 \%$ of $\alpha$-methylnaphthalene was injected through an injector to match the refractive index of the liquid with that of acrylic, by which we can clearly observe cavitation in the injector and accurately measure the liquid velocity distribution in the sac. Note that the outer surfaces of the injectors are perpendicular to the optical axis. As shown in Figure $1 \mathrm{a}$, a high-speed camera (Photron, FASTCAM SA-Z, 2500-5000 fps, exposure time $=10 \mu \mathrm{s}$, $1280 \times 504$ pixels or $640 \times 1024$ pixels) and a metal halide light (KYOWA co., Itd., MID-25FC, $250 \mathrm{~W}$ ) were used for high-speed imaging.

To measure unsteady velocity distributions in the sacs, high-speed stereo PIV or high-speed PIV analyses were carried out. Figure $1 \mathbf{b}$ shows the experimental setup for high-speed PIV, where a pair of high-speed cameras (Photron, FASTCAM SA-Z, $20000 \mathrm{fps}$, exposure time = $50 \mathrm{~ms}, 1000 \times 1000$ pixels, $10 \mathrm{~mm} /$ pixels) and a mesa PIV laser (AMPLITUDE JAPAN, sheet light thickness $=1 \mathrm{~mm}$ ) were used. While High-speed PIV was conducted to simply measure a transient velocity distribution across a string cavitation in the sac with a long needle tip by using a high-speed camera (Photron, FASTCAM SA-Z, $20000 \mathrm{fps}$, exposure time $=10 \mathrm{~ms}$, 1024 x 1024 pixels) and a laser sheet generator as showed in Figure 1 c. The fluorescent particles (FLUOSTAR, model 0459, $15 \mu \mathrm{m}$ in diameter) were used as tracer particles for all PIV experiments. The stokes number is much smaller than 1 , so that the particles follow the flow lines. A commercial PIV software (FtrPIV, ver. 3.2.3.2, Flowtech Research Inc.) was used for PIV analyses. The more details of the experimental setup and method were described in our previous papers [11][13].

The schematics of transparent three-hole injectors are showed in Figure 2. All the injectors have three orifices, whose diameters were hand finished so that the error among three liquid flow rates from the orifices was less than $1 \%$. We used various injectors with different needle lifts $Z_{i n}$, nozzle hole diameters $D_{N o}$, nozzle hole positions, and needle tip angles $\theta_{N e}$. The dimensions of the injectors are summarized in Table 1. The sac diameter $D_{s}$ was $10 \mathrm{~mm}$ and the orifice length $L_{\text {No }}$ was $8 \mathrm{~mm}$ for all the enlarged injectors. The injectors with various orifice positions (nozzle hole angles $\theta_{N o}$ ), needle lifts $Z_{\text {in }}$, needle tip angles $\theta_{N e}$, orifice diameters $D_{N o}$ were tested. By changing these injector shape factors, the contraction coefficient $C_{C}$ of the 
orifice was varied. Table 2 shows the experimental conditions. The liquid temperature was adjusted by using a heater in the liquid tank.
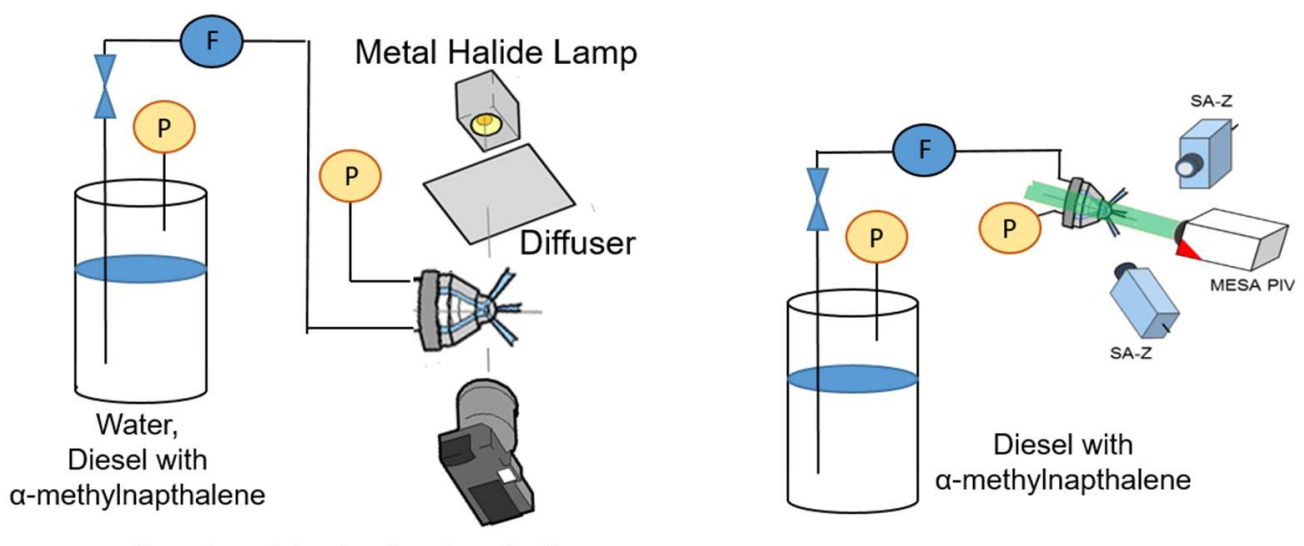

a. Experimental setup for visualization

b. Experimental setup for high-speed stereo PIV
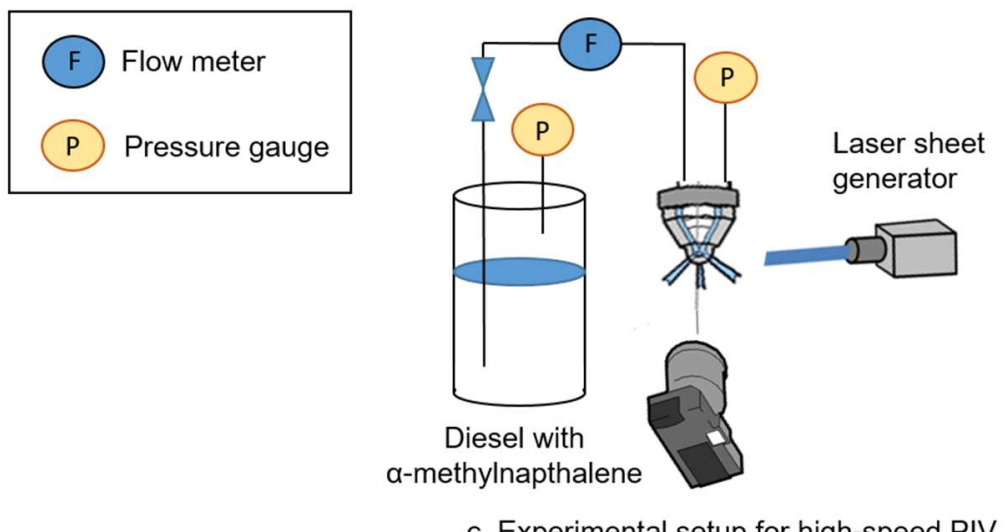

Figure 1. Experimental setup
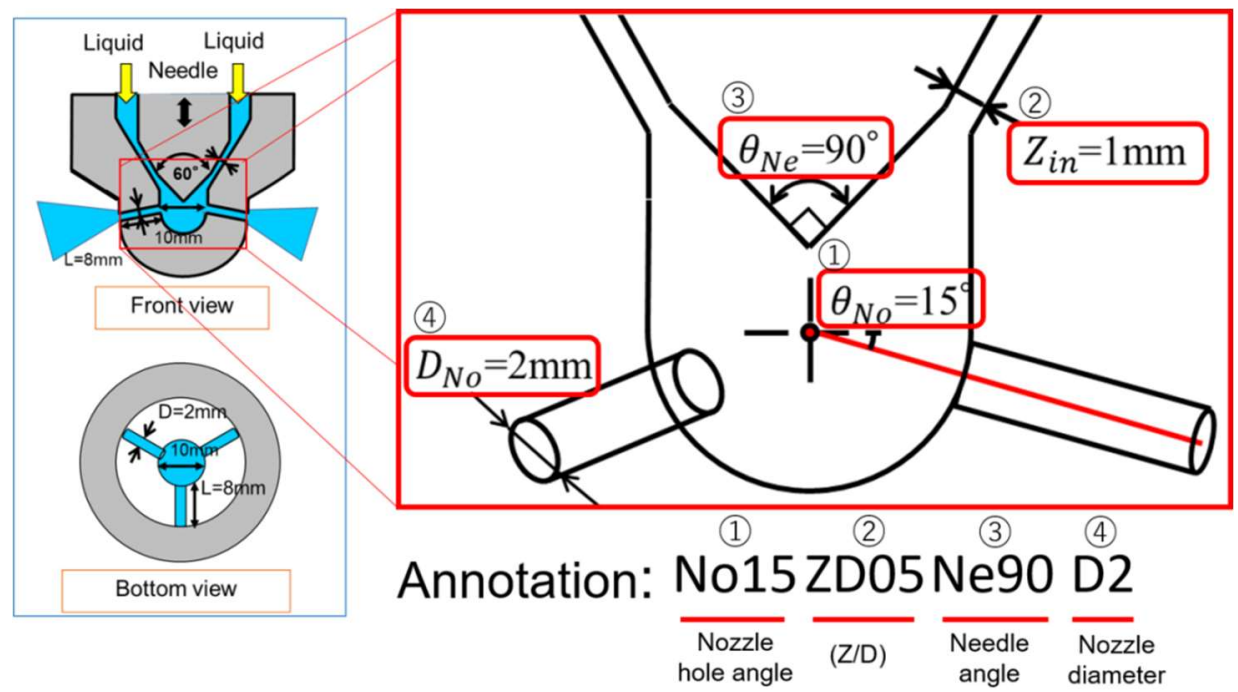

Figure 2. Injector schematics 
Table 1 - Injector dimensions and obtained $C_{c} \& D_{V}$

\begin{tabular}{c|cccccc}
\hline Name & $\begin{array}{c}\theta_{N o} \\
{[\mathrm{deg} .]}\end{array}$ & $\begin{array}{c}Z_{\text {in }} / D_{\text {No }} \\
{[-]}\end{array}$ & $\begin{array}{c}\theta_{N e} \\
{[\mathrm{deg} .]}\end{array}$ & $\begin{array}{c}D_{N o} \\
{[\mathrm{~mm}]}\end{array}$ & $\begin{array}{c}C_{C} \\
{[-]}\end{array}$ & $\begin{array}{c}D_{V} \\
{[\mathrm{~mm}]}\end{array}$ \\
\hline No15ZD05Ne90D2 & 15 & 0.5 & 90 & 2.0 & 0.65 & 2.8 \\
No15ZD3Ne90D2 & 15 & 3.0 & 90 & 2.0 & 0.61 & 0.35 \\
No15ZD025Ne90D2 & 15 & 0.25 & 90 & 2.0 & 0.65 & 2.8 \\
No15ZD05Ne90D1 & 15 & 0.5 & 90 & 1.0 & 0.61 & 0.35 \\
No15ZD05Ne60D2 & 15 & 0.5 & 90 & 2.0 & 0.65 & 0.35 \\
No15ZD1Ne60D2 & 15 & 1.0 & 90 & 2.0 & 0.65 & 0.35 \\
No15ZD3Ne60D2 & 15 & 3.0 & 60 & 2.0 & 0.61 & 0.35 \\
No45ZD05Ne90D2 & 45 & 0.5 & 90 & 2.0 & 0.61 & 0.15 \\
No45ZD3Ne90D2 & 45 & 3.0 & 90 & 2.0 & 0.61 & 0.35
\end{tabular}

Table 2 - Experimental conditions

\begin{tabular}{c|c}
\hline Item & Value \\
\hline Nozzle diameter $D_{N_{0}}[\mathrm{~mm}]$ & $2.0,1.0$ \\
Needle seat gap $Z_{\text {in }}[\mathrm{mm}]$ & $1.0,6.0\left(Z_{\text {in }} / D_{N o}=0.5,3.0\right)$ \\
Nozzle length $L_{N o}[\mathrm{~mm}]$ & $8.0(L / D=4.0,8.0)$ \\
Mean velocity in nozzle $V_{N o}[\mathrm{~m} / \mathrm{s}]$ & $10.0-17.0$ \\
Injection Pressure $P[\mathrm{MPa}]$ & $0.19,0.23$ \\
Liquid & Diesel oil $(79 \%)$ with $\alpha$-methyl \\
Temperature $T[\mathrm{C}]$ & naphthalene $(21 \%)$, water \\
Density $\rho\left[\mathrm{kg} / \mathrm{m}^{3}\right]$ & $31 \pm 1$ \\
& 845
\end{tabular}

\subsection{Numerical model}

Numerical simulation was performed using CONVERGE (version 2.3.17, IDAJ Co., LTD) to examine the transient velocity and cavitation distributions in the sac and orifices. We carried out some numerical simulations with different orifice numbers, injector sizes, needle lifts, and liquid velocities to investigate their effects on the volume fraction of cavitation in the sac and orifices. As shown in Figure 3, the simulation was performed for the 1/6 region of the threehole injectors and the $1 / 12$ region of the six-hole injector by assuming symmetric flow. The geometries of the injectors simulated were the same as those of the transparent injectors used in the experiments, whose needle tip angle was 90 degrees and orifice angle was 15 degrees. The RNG $k-\varepsilon$ model was used as the turbulence model. The simplified Rayleigh-Plesset equation was solved to simulate cavitation growth and collapse, which was used to evaluate the source term of the transport equation of cavitation bubbles. The minimum cell size was set to $63 \mu \mathrm{m}$ as a result of sensitivity analysis and the courant number is 0.1 . The total number of computational cells for $1 / 6$ region of the three-hole injectors was about 180.000 . The mesh was determined based on mesh sensitivity analysis. The simulation conditions are showed in Table 3. 

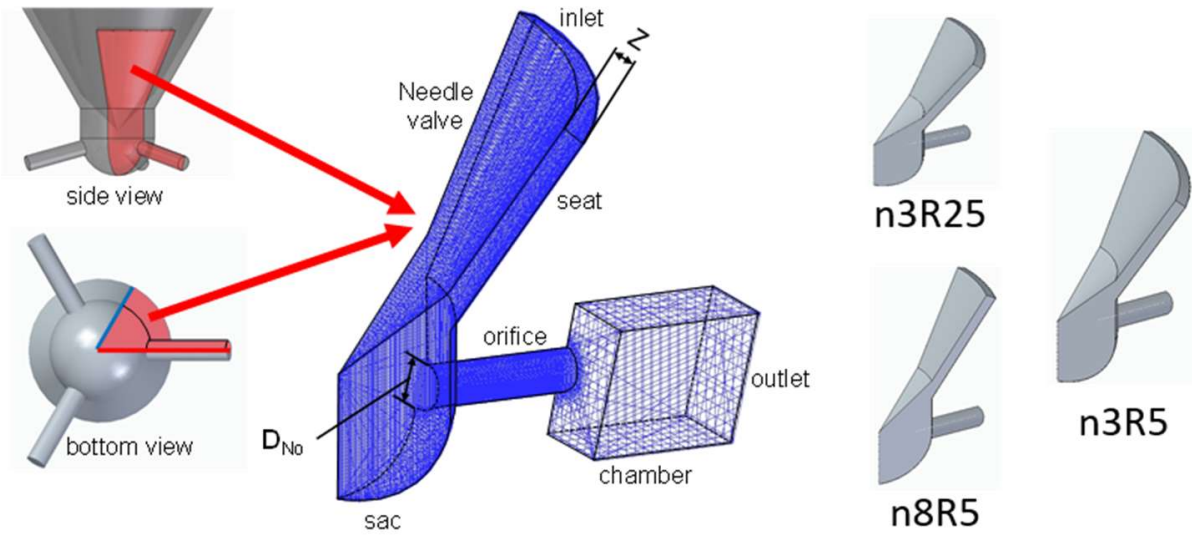

Figure 3. Simulation systems of three-hole and eight-hole injectors

Table 3 - Conditions of numerical simulation

\begin{tabular}{|c|c|c|c|c|c|c|c|c|c|}
\hline Case & $\begin{array}{c}\text { n3V16 } \\
\text { R5 }\end{array}$ & $\begin{array}{c}\text { n6V8 } \\
\text { R5 }\end{array}$ & $\begin{array}{c}\text { n6V16 } \\
\text { R5 }\end{array}$ & $\begin{array}{c}\text { n6V32 } \\
\text { R5 }\end{array}$ & $\begin{array}{c}\mathrm{n} 8 \mathrm{~V} 6 \\
\mathrm{R} 5\end{array}$ & $\begin{array}{c}\text { n8V16 } \\
\text { R5 }\end{array}$ & $\begin{array}{l}\text { n8V43 } \\
\text { R5 }\end{array}$ & $\begin{array}{c}\text { n3V16 } \\
\text { R25 }\end{array}$ & $\begin{array}{c}\text { n3V32 } \\
\text { R25 }\end{array}$ \\
\hline $\begin{array}{c}\text { Nozzle number } \\
n_{N}\end{array}$ & 3 & 6 & 6 & 6 & 8 & 8 & 8 & 3 & 3 \\
\hline $\begin{array}{l}\text { Mean velocity } \\
\text { at sac inlet } V_{\text {sin }} \\
{[\mathrm{m} / \mathrm{s}]}\end{array}$ & 4.1 & 4.1 & 8.0 & 16 & 4.1 & 11 & 28 & 4.1 & 8.2 \\
\hline $\begin{array}{c}\text { Mean velocity } \\
\text { in nozzle } V_{\text {No }} \\
{[\mathrm{m} / \mathrm{s}]}\end{array}$ & 16 & 8 & 16 & 32 & 6 & 16 & 43 & 16 & 32 \\
\hline$Z_{\text {in }}[\mathrm{mm}]$ & 1.0 & 1.0 & 1.0 & 1.0 & 1.0 & 1.0 & 1.0 & 0.5 & 0.5 \\
\hline $\mathrm{D}_{\mathrm{No}}[\mathrm{mm}]$ & 2.0 & 2.0 & 2.0 & 2.0 & 2.0 & 2.0 & 2.0 & 1.0 & 1.0 \\
\hline$Z_{\text {in }} / D_{\text {No }}$ & 0.5 & 0.5 & 0.5 & 0.5 & 0.5 & 0.5 & 0.5 & 0.5 & 0.5 \\
\hline $\mathrm{W}_{\mathrm{S}}[\mathrm{mm}]$ & 8.7 & 5.0 & 5.0 & 5.0 & 3.8 & 3.8 & 3.8 & 4.3 & 14.3 \\
\hline $\mathrm{Rs}_{\mathrm{s}}[\mathrm{mm}]$ & 5.0 & 5.0 & 5.0 & 5.0 & 5.0 & 5.0 & 5.0 & 2.5 & 2.5 \\
\hline$\Delta \mathrm{x}_{\mathrm{N}}[\mu \mathrm{m}]$ & 62.5 & 62.5 & 62.5 & 62.5 & 62.5 & 62.5 & 62.5 & 31.3 & 31.3 \\
\hline
\end{tabular}

\section{Results and Discussion}

\subsection{High-speed imaging}

Figure 4 a-c shows some images of cavitation in the injectors and discharged liquid jets. For all the cases, no cavitation (NC) can be found at very low injection pressure and low flow rate. When we increase liquid velocity $V_{N_{0}}$ an orifice up to $12-17 \mathrm{~m} / \mathrm{s}$, incipient cavitation (IC) occurs at the vena contracta near the inlet of an orifice. At a higher liquid velocity, super cavitation (SP) is developed with a resulting large angle of a largely deformed liquid jet. At much larger velocity, the discharged liquid jet finally becomes a total hydraulic flip (THF) [4] in the cases with a very high needle lift, and it becomes imperfect hydraulic flip (IHF) with an atomized liquid jet at low needle lift [11]. When the length-to-diameter ratio $L_{N o} / D_{N_{N}}$ of the orifice is large $\left(L_{N o} / D_{N o}=8\right)$, the jet angle is smaller than those with smaller $L_{N o} / D_{N_{0}}$ of 4 [6]. As shown in Figure $4 \mathbf{~ b}$, in case No15ZD05Ne90D2, string cavitation appears randomly, which connects two neighbouring orifices. It should be noted that the number of this type of string cavitation appearing in the sac is zero, one, two, or three [11]. We will call it twin string cavitation in the following, where at most two string cavitations may take place from one orifice to the neighbouring two orifices. When twin string cavitation takes place, the spray angle becomes 
very large, and the discharged liquid becomes a hollow-cone spray. In case No45ZD05Ne90D2 three orifices are located near the bottom of the sac, and twin string cavitation with smaller diameter and shorter length appears rarely. As a result, the spray angle is relatively small. As shown in Figure 4 c, in case No15ZD05Ne60D2 with low needle lift and a long needle tip whose surface is close to the orifice entrance, two steady string cavitations with very large diameters appear in the injector. Since only single string cavitation appears from an orifice to the needle tip surface, we will call it single string cavitation in the following. When single string cavitation takes place, the angle of the discharged hollow-cone spray becomes very large.

\subsection{Measurement of velocity distribution by high-speed stereo PIV and high-speed PIV}

Figure $\mathbf{5}$ shows the velocity distribution and flow patterns in the sac obtained by high-speed stereo PIV, where $D_{v}$ is the vortex diameter whose values for all the cases are shown in Table 1. When needle lift is low, needle tip is far from the orifice entrance and the orifices are near the sac inlet (No15ZD05Ne90D2), $D_{v}$ is very large with possible twin string cavitation. When the orifices are near the sac bottom (No45ZD05Ne90D2), $D_{v}$ is small. This is the reason for the rare and small string cavitation. When needle lift is very high, flow in the sac is almost uniformly downward, which results in no string cavitation.

Figure 6 shows the velocity profile in case No15ZD05Ne60D2 obtained by high-speed PIV when mean liquid velocity $V_{N_{0}}$ in an orifice is $11 \mathrm{~m} / \mathrm{s}$. It shows the results in the planes 2 and $3 \mathrm{~mm}$ from the orifice inlet in the cases with a single string cavitation. From these results, we can confirm that a very large, strong and steady circulation flow is formed around a single string cavitation. Since the orifice number is odd number, the circulation flow with single string cavitation is formed for two orifices. Note that the two orifices with single string cavitation and the circulation flow are randomly determined for each injection. Once they take place in the two orifices, they are steady until the end of injection. The flow pattern and single string cavitation are formed by the bathtub effect, when a lateral inflow along with the thin seat (low needle lift) and sac wall does not quickly change its direction toward the orifice axis but forms a circulation flow. Measured circulation in the sac is shown in Figure 7. We can clearly confirm that the magnitude of the circulation at the right half of the sac is almost as large as that at the left half, and they are in opposite directions in the case with twin string cavitation, the resulting spiral flow at the exit of the orifice becomes weak. On the other hand in the case with single string cavitation induced by long needle tip, the steady, large and strong single circulation flow reaches the orifice exit and induces a hollow-cone spray by the strong centrifugal force.

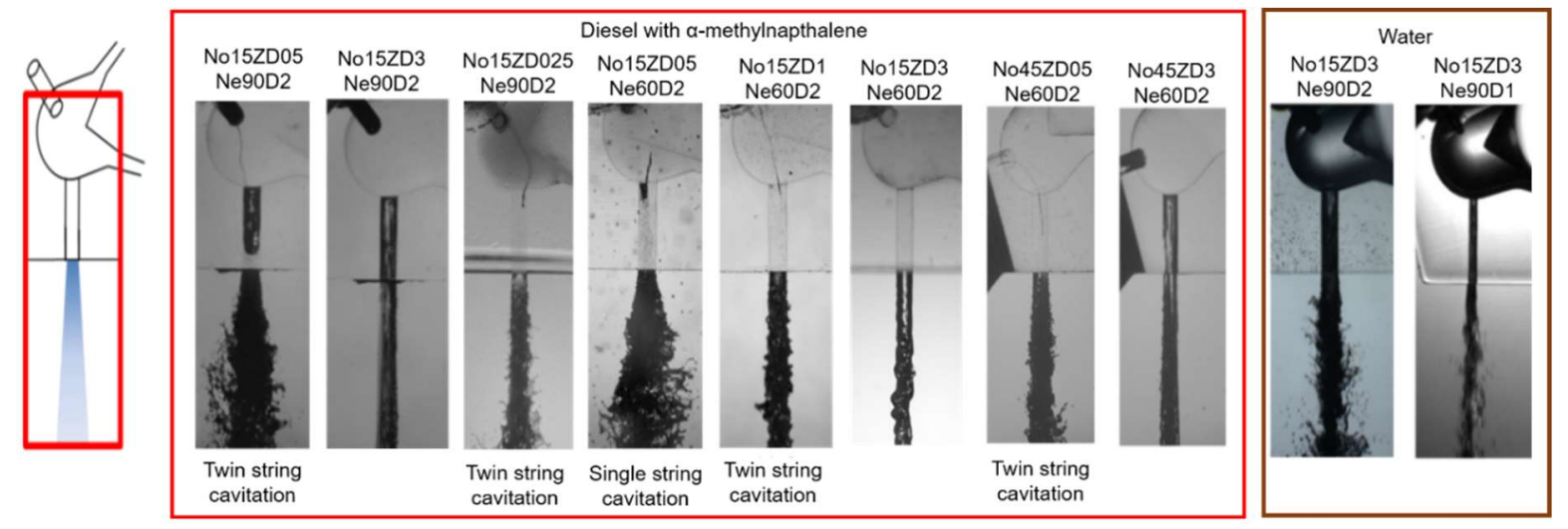

(a) Cavitation in the orifice and discharged liquid jet

Figure 4. Sample images of cavitation in the injectors and discharged liquid jets (to be continued) 


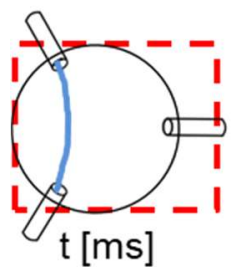

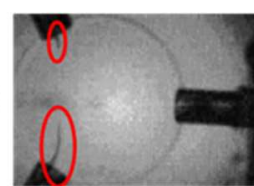

0.00

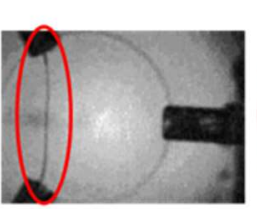

0.33

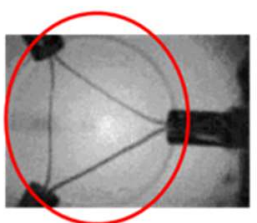

1.00

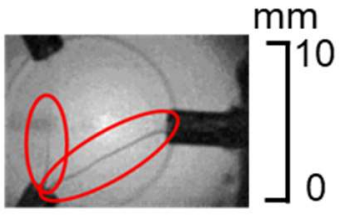

1.67

(b) Bottom view of twin string cavitation (No15ZD05Ne90D2)

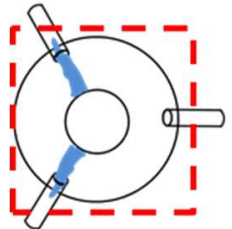

$\mathrm{t}[\mathrm{ms}]$

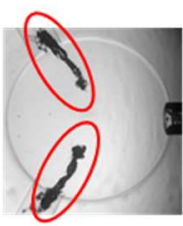

0.00

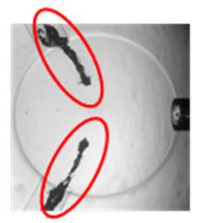

0.33

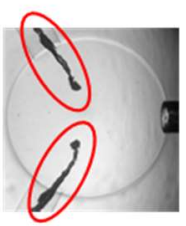

1.00

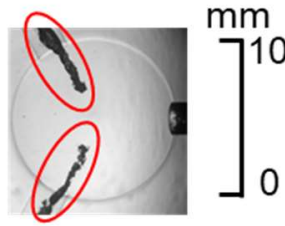

1.67

(c) Bottom view of single string cavitation (No15ZD05Ne60D2)

Figure 4. Sample images of cavitation in the injectors and discharged liquid jets (continued)

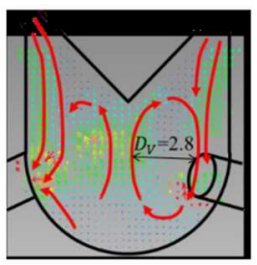

(a) No15ZD05Ne90D2

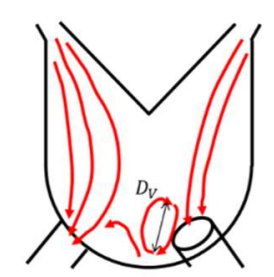

(b) No45ZD05Ne90D2

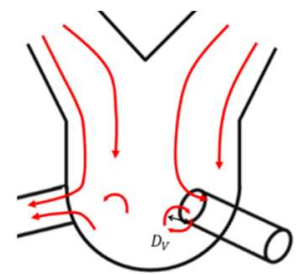

(c) No15ZD3Ne90D2

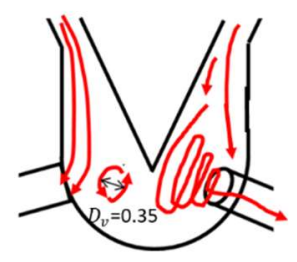

(d) No15ZD05Ne60D2

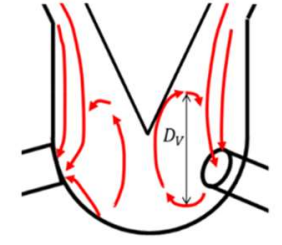

(e) No15ZD1Ne60D2

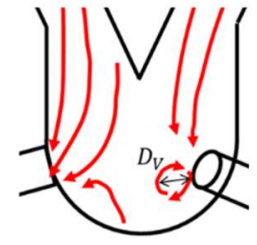

(f) No15ZD3Ne60D2

Figure 5. Flow patterns in the mini-sac fuel injectors based on the visualization and measurement

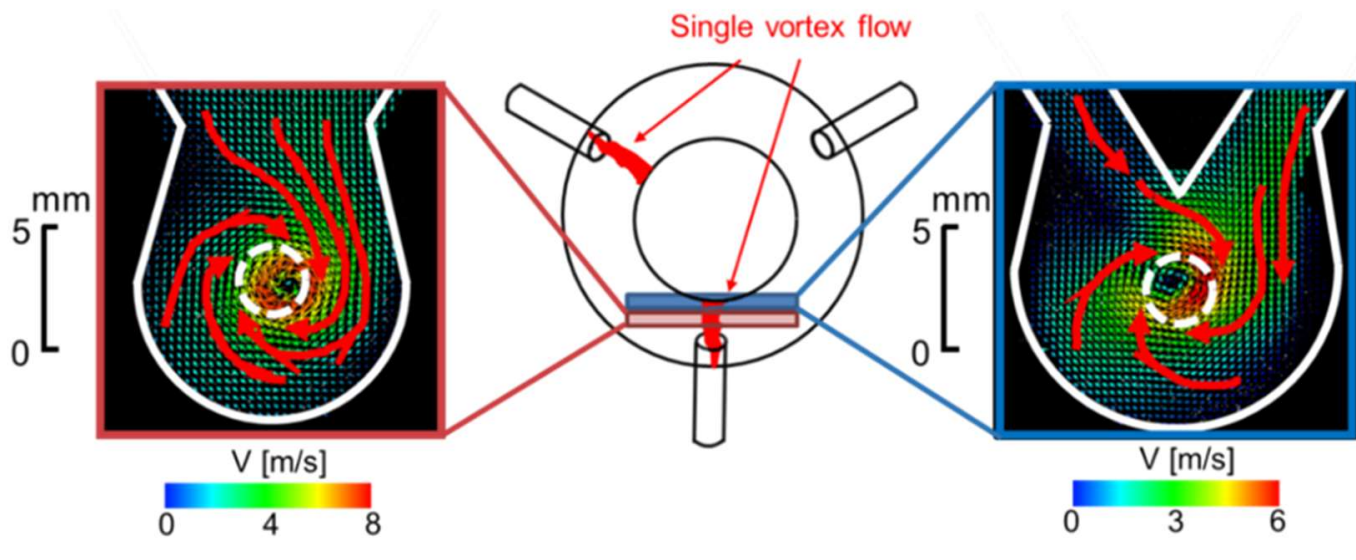

Sheet distance $2 \mathrm{~mm}$

Bottom view

Sheet distance $3 \mathrm{~mm}$

Figure 6. Measured velocity distribution obtained by High-speed PIV 


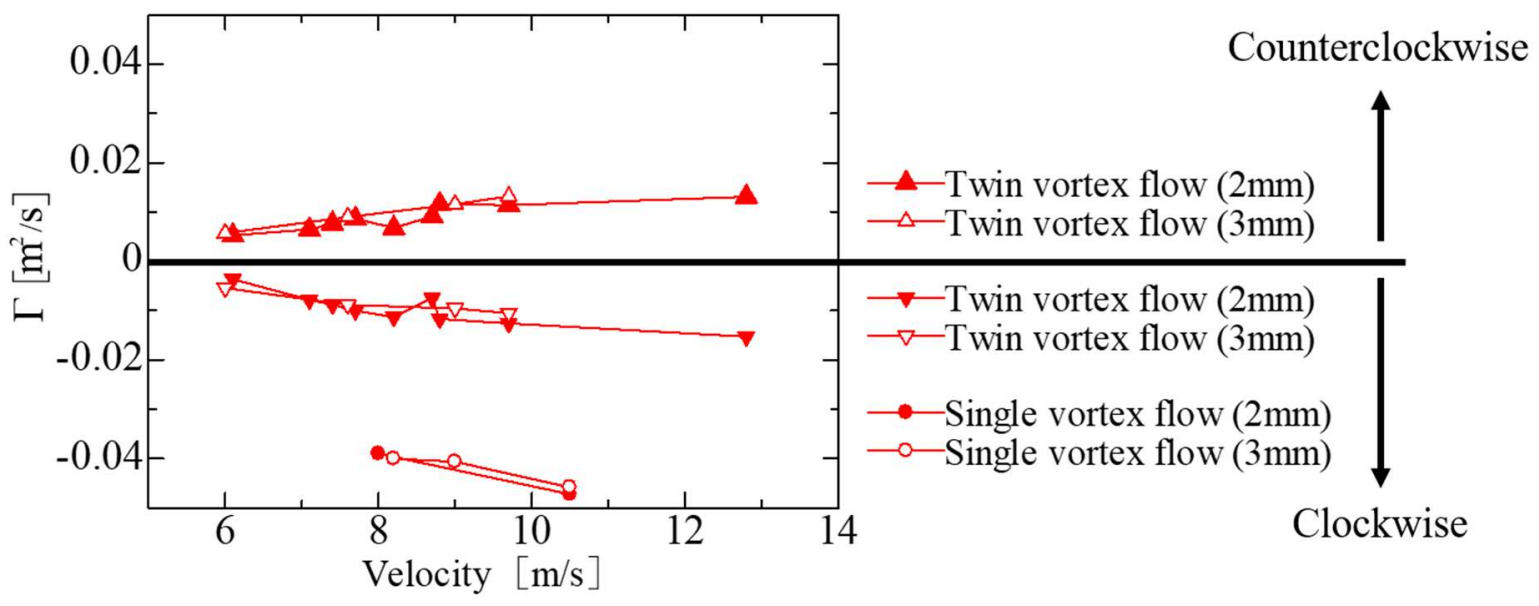

Figure 7. Measured circulation in the sac with single and twin string cavitation

\subsection{Numerical simulation}

Figure 8 shows some simulated distributions of velocity and cavitation volume fraction in the injectors with different orifice numbers, injector sizes, and liquid velocities. A strong croissantshaped vortex structure is formed between two neighbouring orifices, and twin string cavitation appears when orifice number is small, liquid velocity is large, sac radius is large, and needle lift is small ( $n 3 V 16 R 5$, orifice number $n=3$, liquid velocity $V_{N_{0}}=16 \mathrm{~m} / \mathrm{s}$, sac radius $R s=5 \mathrm{~mm}$, and needle lift $Z_{\text {in }} / D_{N_{0}}=0.5$ ). These results agree with the measured velocity distributions and observed cavitation pattern, by which we can confirm the above-mentioned flow mechanisms.

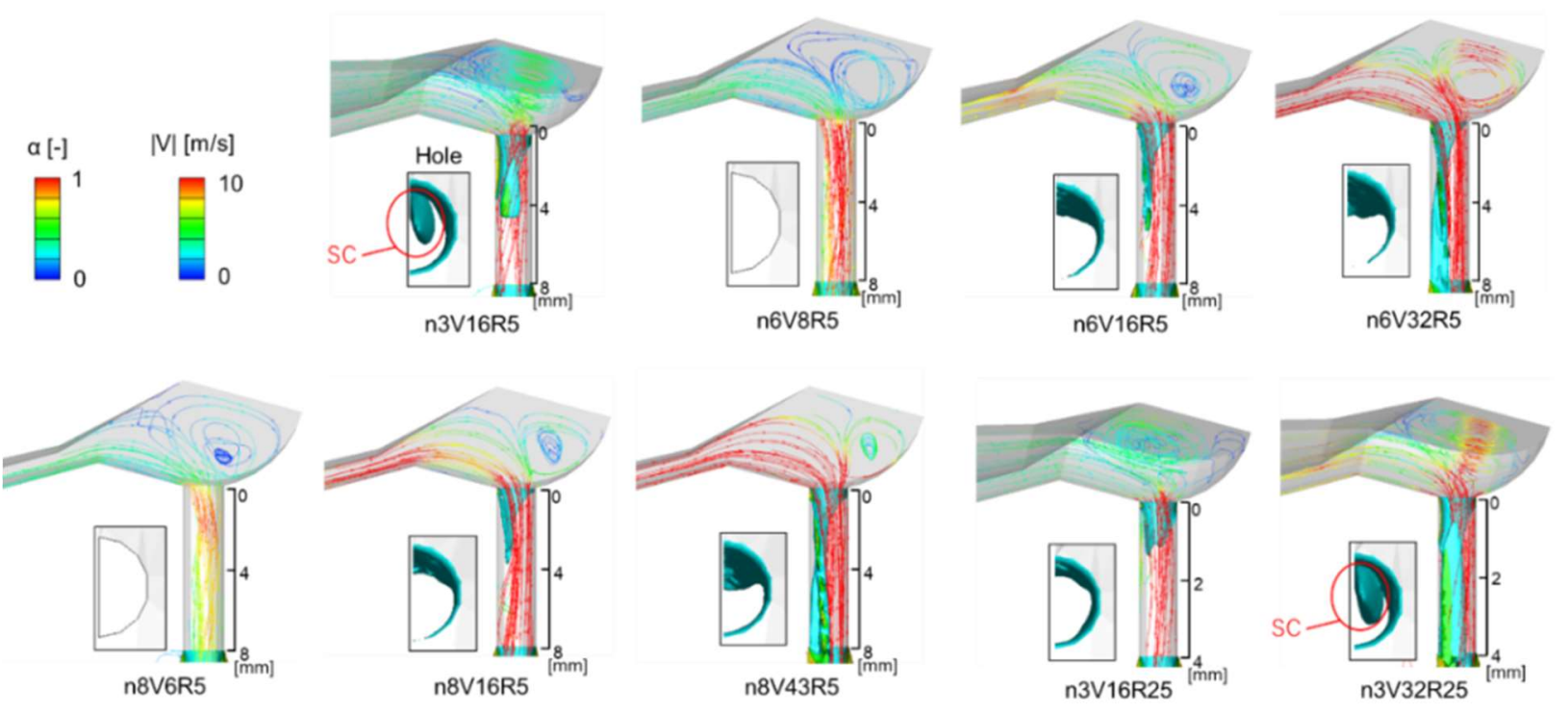

Figure 8. Simulated velocity and cavitation distributions in various injectors

3.4 Dimensionless indices governing turbulent cavitation flow in mini-sac nozzles Atomization of spray is enhanced at super cavitation (SP) and imperfect hydraulic flip (IHF) with long cavitation along the orifice wall. To quantitatively predict cavitation length in an orifice, we have confirmed that a dimensionless index, the modified cavitation number $\sigma_{c}$ based on 
the local pressure at vena contracta of the orifice, can be used to quantitatively predict cavitation length, cavitation inception and super cavitation formation, which is defined by [5][6]:

$$
\sigma_{C}=C_{C}{ }^{2}\left[\frac{P_{b}-P_{v}}{\frac{1}{2} \rho V_{N o}{ }^{2}}+\frac{\lambda L_{N o}}{D_{N o}}+1\right]
$$

where $P_{b}$ is the atmospheric pressure, $P_{v}$ is the saturated vapor pressure, $\rho$ is the liquid density, $\lambda$ is the friction factor. The $C_{c}$ represents the contraction coefficient which is defined by

$$
C_{C}=\frac{S_{C}}{S_{N o}}
$$

where $S_{N_{0}}$ denotes the cross-sectional area of the orifice, and $S_{c}$ is that of the core flow. The value of $C_{c}$ is 0.61 for the single-hole nozzle with orifices far from the needle seat or with high needle lift, while the value of $C_{c}$ is found to be 0.65 based on numerical velocity distribution for the cases with low needle lift. The value of $C_{c}$ is resumed in Table 1.

The dimensionless index $\operatorname{Re}_{s}$ which governs the toroidal vortex flow with twin string cavitation should take the following form.

$$
R e_{S}=\frac{\rho V_{S}^{2}}{\mu \frac{V_{S}}{L_{S}}}=\frac{V_{S} L_{s}}{v}
$$

where $v$ is the kinematic viscosity. The representative velocity $V_{S}$ and the representative length $L_{s}$ are given by the following equations.

$$
\begin{aligned}
& V_{S}=\max \left\{\left(\frac{D_{S}}{Z_{\text {in }}}\right) V_{\text {in }},\left(\frac{D_{N o}}{D_{S}}\right) V_{N o}\right\} \\
& L_{S}=\min \left\{W_{S}, D_{V}, Z_{N o}\right\}
\end{aligned}
$$

The representative velocity $V_{S}$ is given as the maximum of the velocity $V_{\text {in }}$ flowing into the sac through the seat and the velocity $V_{\text {No }}$ flowing out to a nozzle hole. As illustrated in Figure 9, the representative length $L_{s}$ is given as the minimum of the distance $W_{s}$ between two neighbouring orifices, the diameter $D_{v}$ of the vortex in the sac (see Table 1), and the distance $Z_{\text {No }}$ between the orifice inlet and the needle surface.

In the case of single string cavitation with long needle tip whose surface is close to the orifice entrance, the dimensionless index $R e_{1}$ is given by

$$
R e_{1}=\frac{\rho V_{N o}^{2}}{\mu \frac{V_{N o}}{D_{N o}}} \frac{\mu \frac{V_{N o}}{Z_{N o}}}{\mu \frac{V_{N o}}{W_{S}}} \sin \theta_{1}=\frac{D_{N o} W_{S} V_{N o}}{v Z_{N o}} \sin \theta_{1}
$$

The validity of the dimensionless index $R e_{1}$ is confirmed through a visualization experiment using a lot of simple rectangular injectors with various $W_{S}, V_{N_{0}}$ and $Z_{N_{0}}[10]$. 


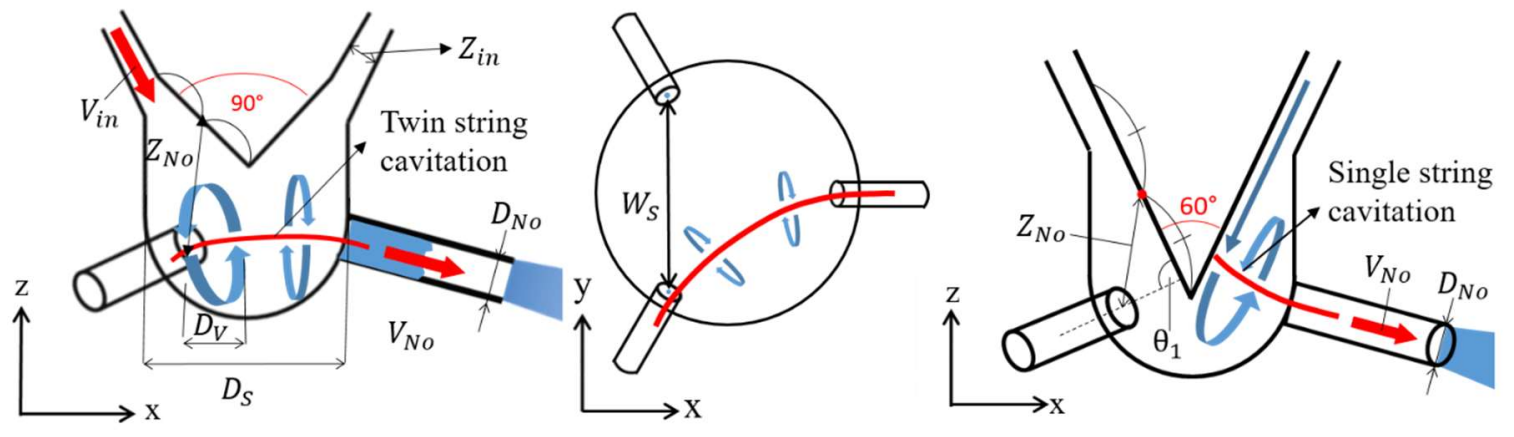

Figure 9. Characteristic velocity and length of the flow in mini-sac injectors

3.5 Applicability of the dimensionless indices to the prediction of cavitation flow and spray Figure 10 a shows the relation between measured cavitation length $L_{C}$ in an orifice and liquid velocity $V_{N_{0}}$ in the orifice. We can clearly confirm that cavitation takes place and is developed at larger $V_{N o}$ when the orifices are located near the sac bottom and contraction coefficient is smaller, while cavitation occurs at smaller $V_{\text {No }}$ when water with larger density is injected. Hence, we cannot predict cavitation in an orifice quantitatively only based on liquid velocity $V_{N_{0}}$ or liquid flow rate. Figure $\mathbf{1 0} \mathbf{b}$ shows the relation between cavitation length $L_{c}$ and modified cavitation number $\sigma_{c}$. The result confirms that by using modified cavitation number $\sigma_{c}$ as the dimensionless index we can predict quantitatively the cavitation length for various injectors with different injector geometries, orifice positions, needle lifts, orifice diameters, needle tip geometries and liquid properties, that is $L_{d} L=0.1-0.4$ at $\sigma_{c}=1$ (cavitation inception) and $L_{C} L=0.7-1$ at $\sigma_{c}=0.7-0.8$ (super cavitation or IHF). As shown in Figure $10 \mathrm{c}$, measured spray angle $\theta$ is small in the cases of large length-to-diameter ratio $\left(L / D_{N_{0}}=8\right)$ or water with large surface tension, and it becomes very large in the cases with twin string cavitation and extremely large in the cases with single string cavitation. That is why we cannot quantitatively predict spray angle $\theta$ based only on $L_{d} L$. However, the effect of super cavitation and IHF to increase spray angle $\theta$ can be estimated by knowing $L d L$ for the cases without string cavitation. As a result, the increase in spray angle $\theta$ by super cavitation and IHF can be predicted well by using modified cavitation number $\sigma_{c}$, as shown in Figure $10 \mathrm{~d}$.

Figure 11 a shows the relation between measured spray angle $\theta$ and measured circulation $\Gamma$ in the sac. The result confirms that circulation $\Gamma$ in the sac is significantly large when there appears single string cavitation, which results in a significantly large spray cone angle induced by a significantly large centrifugal force. Figure 11 b shows the relation between spray angle $\theta$ and the normalized diameter of string cavitation $D_{S C}$ which is defined by the following equation.

$$
D_{S C}=\frac{S_{S C}}{W_{S} D_{N o}}
$$

Where $S_{s c}$ is the string cavitation area. The result confirms the extremely large spray angle in the cases with single string cavitation (case Diesel-No15ZD05Ne60D2). 


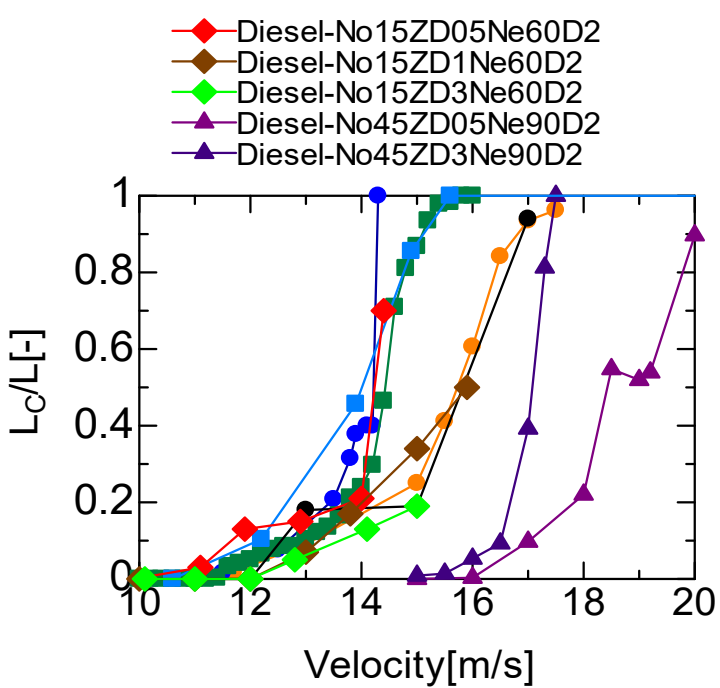

(a) $L_{c} / L$ vs. $V_{\text {No }}$

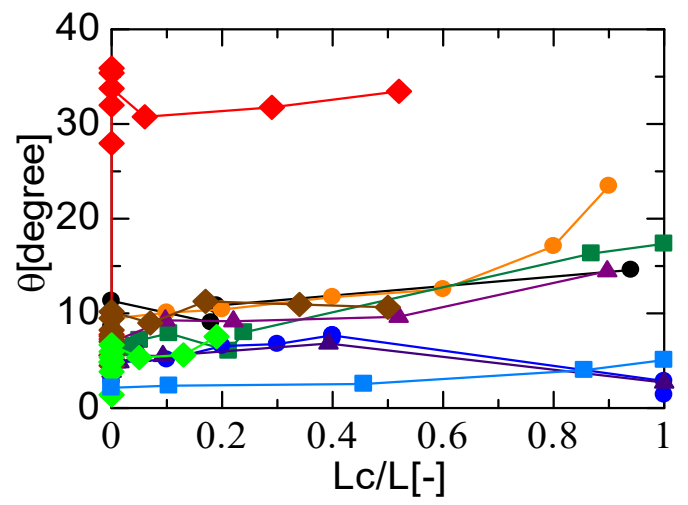

(c) $\theta$ vs. $L_{c} / L$

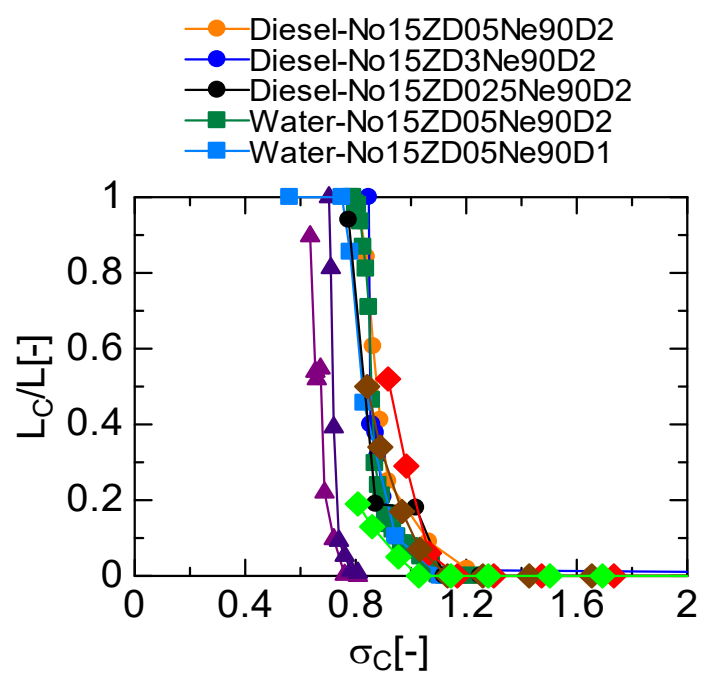

(b) $L_{c} / L$ vs. $\sigma_{c}$

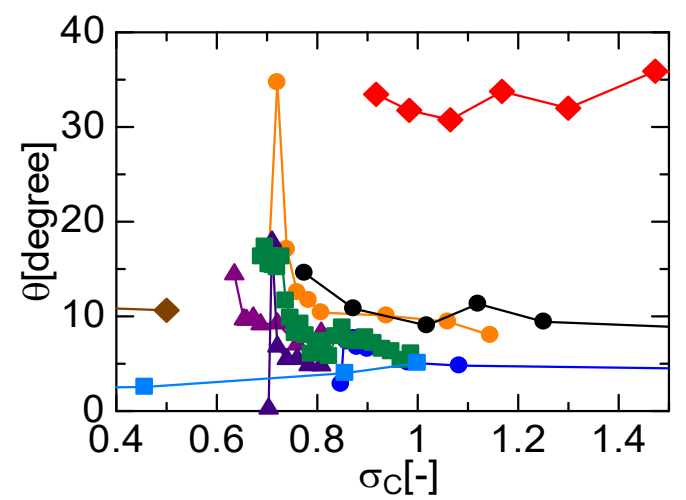

(d) $\theta$ vs. $\sigma_{c}$

Figure 10. Quantitative prediction of cavitation length $L_{c}$ in an orifice and spray angle $\theta$
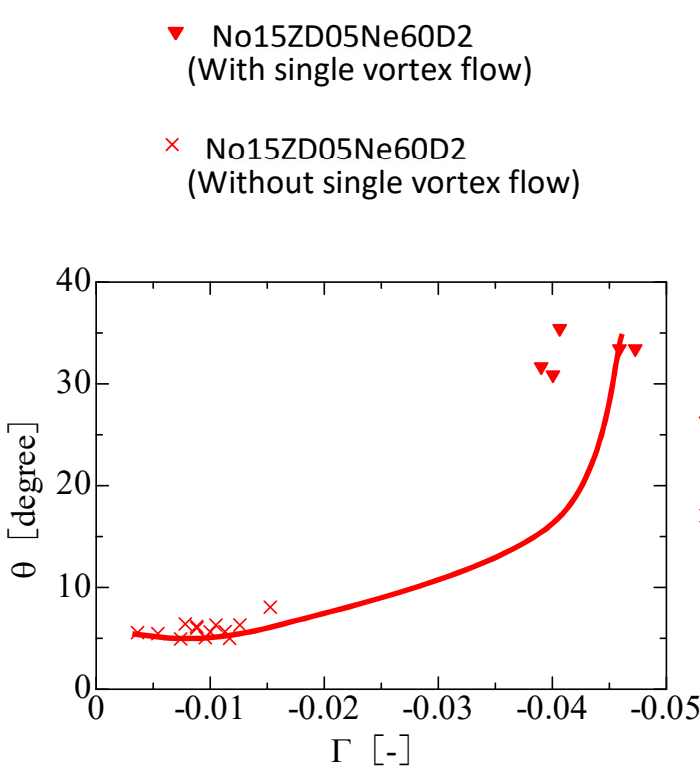

(a) $\theta$ vs. $\Gamma$
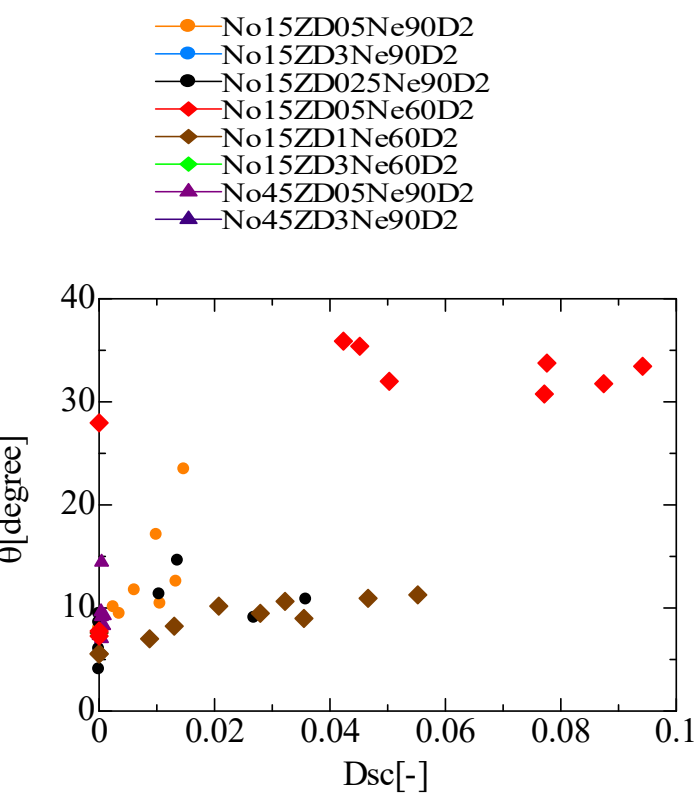

(b) $\theta$ vs. Dsc

Figure 11. Relation between measured spray angle and string cavitation diameter Dsc or circulation $\Gamma$ in the sac 
Figure 12 shows the distribution map of non-cavitation, twin string cavitation and single string cavitation by taking $R e_{1}$ and $R e_{S}$ as dimensionless indices. The map indicates that (1) string cavitation does not appear and super cavitation and IHF are dominant for spray characteristics when $R e_{S}<1800$ and $R e_{1}<8000$, (2) single string cavitation occurs when $R e_{1}>8000$ and $R e_{s}<500$, and (3) twin string cavitation may occur intermittently when $R e_{s}>1400$. It should be noted that these results are based only on the present experiment and numerical simulation of steady injection process using enlarged injectors at small liquid velocity and low injection pressure with fixed needle valve. In the case of transient diesel injection process, we have to take into account the effect of string cavitation on spray at the moment when $R e_{s}>1400$ or $R e_{1}>8000$, and that of super cavitation and IHF when $R e_{S}<1800, R e_{1}<8000$ and $\sigma_{C}<1$.

Figure 13 shows measured circulations $\Gamma$ in the sac plane, which directly increases spray angle, taking $R e_{s}$ or $R e_{1}$ as horizontal axis. The results clearly indicate that $\Gamma$ is small and almost proportional to $R e_{s}$ when there is no single string cavitation, while it takes a significantly large value when there is single string cavitation.

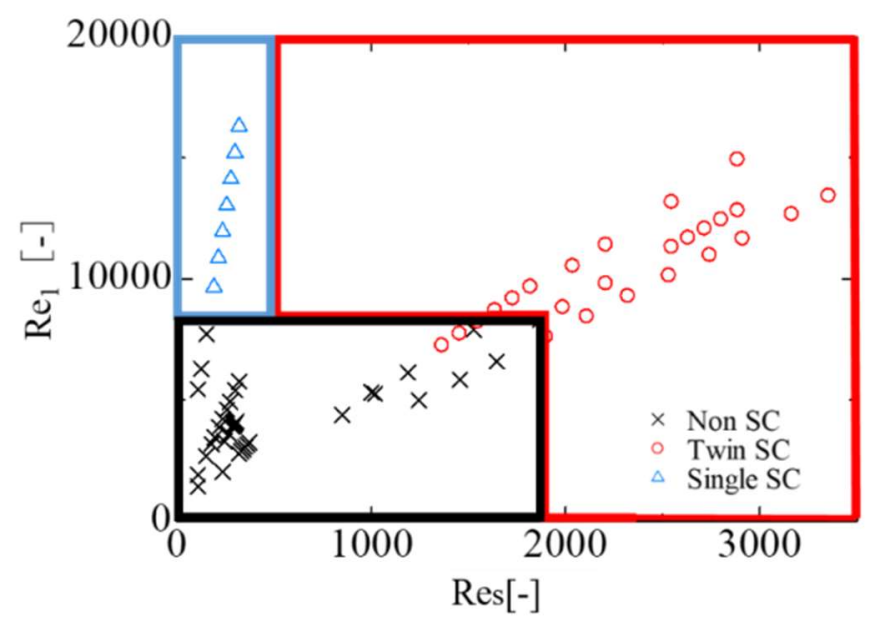

Figure 12. $\left(\operatorname{Re}_{1}, \mathrm{Re}_{\mathrm{s}}\right)$ map for single and twin string cavitation

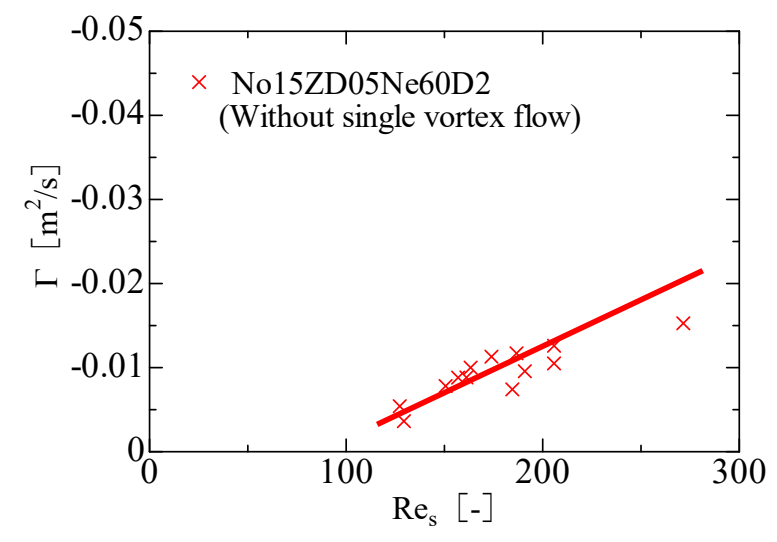

(a) $\Gamma$ vs. Res

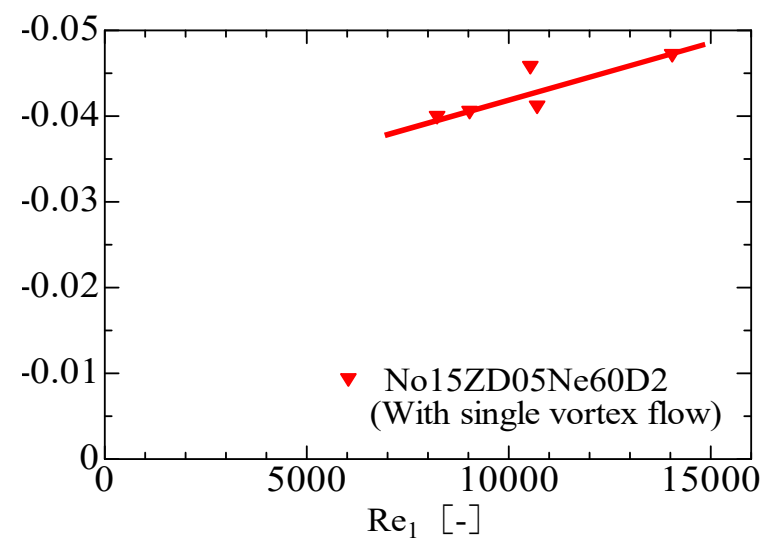

(b) $\Gamma$ vs. $\operatorname{Re}_{1}$

Figure 13. Measured circulation $\Gamma$ in a sac and $\operatorname{Re}_{s}$ or $\operatorname{Re}_{1}$

Figures 14 a-c show measured the normalized diameter of string cavitation $D_{S c}$, which may show a clear relation with spray characteristics. Figure 14 a clearly indicates that it is not 
possible to predict quantitatively string cavitation diameter $D_{S c}$ only based on liquid velocity $V_{N o}$ in an orifice. On the other hand, as shown in Figure $14 \mathbf{b}$, we can confirm that single string cavitation occurs when $R e_{1}>8000$ and its diameter is almost proportional to $R e_{1}$. Finally, as shown in Figure $14 \mathrm{c}$, twin string cavitation may take place intermittently when $R e_{s}>800$, however, the diameter of twin string cavitation seems to be difficult to be predicted quantitatively. In contrast, as shown in Figures $14 \mathrm{~d}$ and e, an extremely large value of spray angle $\theta$ with single string cavitation seems to be appears only when $R e_{1}>8000$, and spray angle $\theta$ with twin string cavitation is proportional to $R e_{s}$. when $R e_{s}>800$.

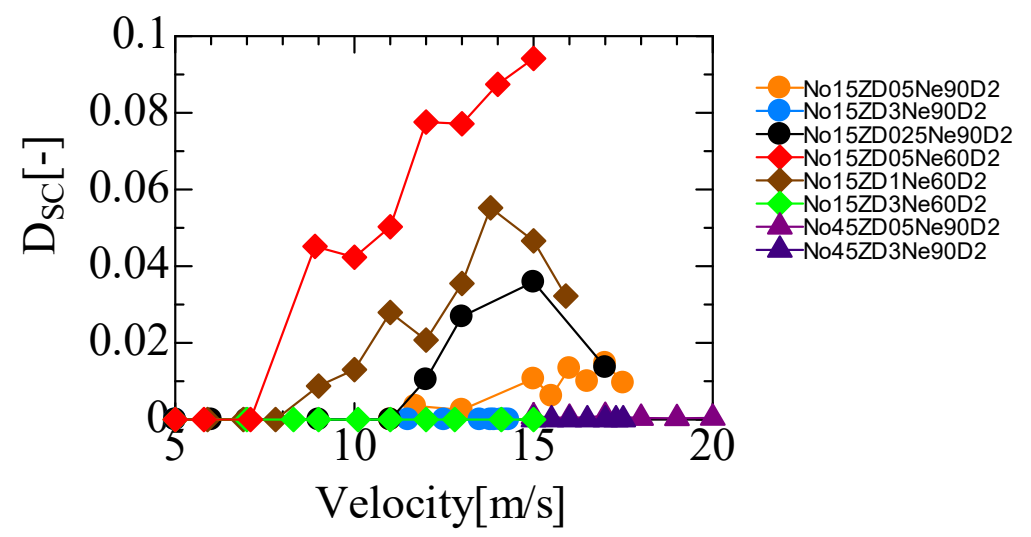

(a) $D_{\text {SC }}$ vs. $V_{\text {No }}$

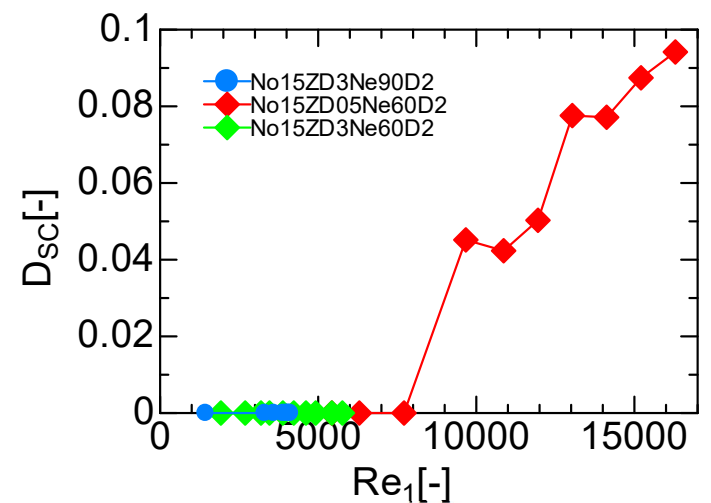

(b) $D_{\text {sC }}$ vs. $\operatorname{Re}_{1}$

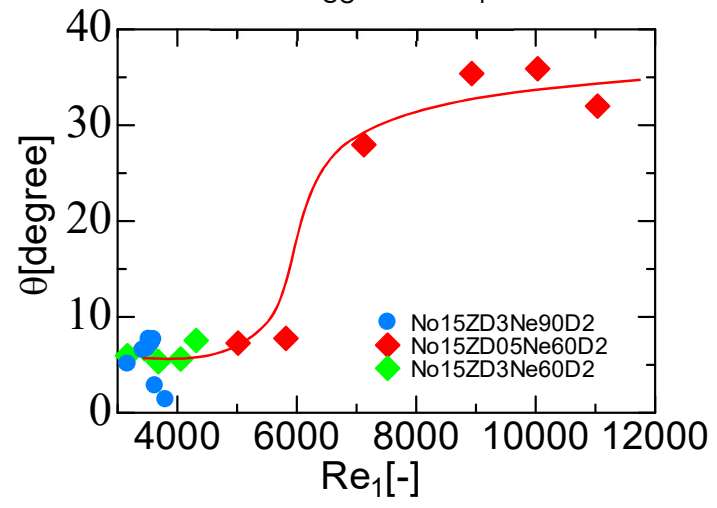

(d) $\theta$ vs. $\operatorname{Re}_{1}$

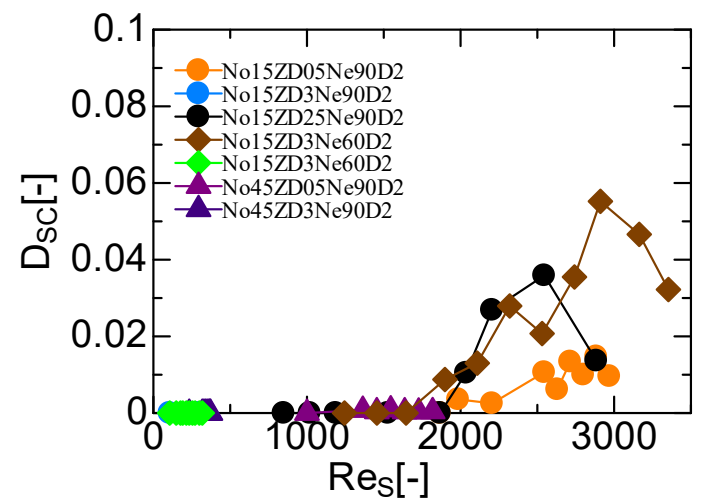

(c) $D_{S C}$ vs. $R e_{S}$

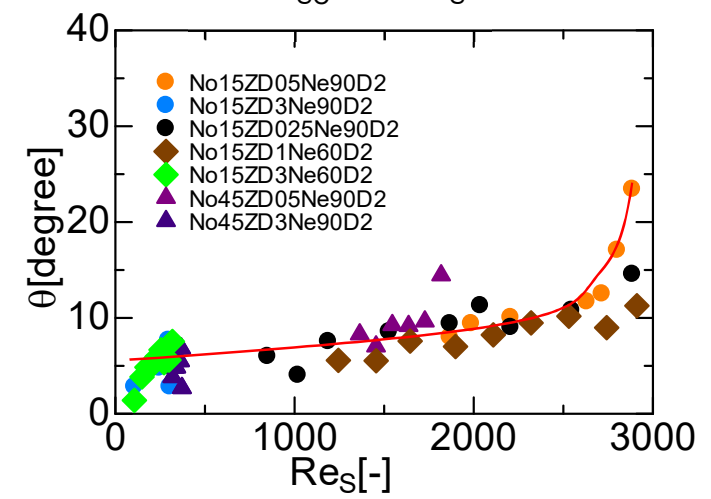

(e) $\theta$ vs. $\operatorname{Re}_{\mathrm{s}}$

Figure 14. Measured string cavitation diameter Dsc and spray angle $\theta$ in the cases with string cavitation 


\section{Conclusions}

In this study, we conducted visualization and measurement experiments as well as numerical simulations of steady cavitation flow in enlarged multi-hole mini-sac injectors with fixed needle valve. Based on the results, we proposed three dimensionless indices, modified cavitation number $\sigma_{c}$, single Reynolds number $R_{1}$, and twin Reynolds number $R e_{s}$, whose applicability to the quantitative prediction of string cavitation diameter, circulation in the sac, and spray angle were examined. As a result, we obtained the following conclusions.

1. There are two types of string cavitation, single string cavitation and twin string cavitation, whose occurrence mechanisms and effects on spray characteristics are totally different.

2. Regardless of the geometry of the diesel fuel injector, needle lift, and fluid properties, if the contraction coefficient is given appropriately, cavitation length in a nozzle hole can be quantitatively predicted using the modified cavitation number.

3. When needle lift is low and there is a large volume in the sac of a multi-hole mini-sac nozzle, twin vortex flow, and the resulting twin string cavitation appears intermittently from an orifice to the neighbouring orifices, which increase spiral flow in the orifice and spray angle. The effects of twin string cavitation can be estimated quantitatively by using the twin Reynolds number $R e_{s}$.

4. When needle lift is low and the surface of the needle valve is close to the orifice inlet, single vortex flow and the resulting single string cavitation with large diameter almost always appear from an orifice to the surface of the needle valve, which significantly increase spiral flow in the orifice and spray angle. The effects of single string cavitation can be estimated quantitatively by using single Reynolds number $R e_{1}$.

5. By using the combination of the three dimensionless indices $\sigma_{c}, R e_{s}$ and $R e_{1}$ proposed in this study, we can quantitatively predict circulation in the sac, cavitation length in an orifice, string cavitation diameter, and the resulting spray angle for multi-hole mini-sac nozzles with various geometries, needle lifts, liquid flow rates, and liquid fluid properties.

\section{Nomenclature}

$\mathrm{C}_{\mathrm{c}} \quad$ contraction coefficient [-]

$\mathrm{L}_{\mathrm{No}} \quad$ nozzle orifice length $[\mathrm{mm}]$

$\mathrm{L}_{\mathrm{s}} \quad$ representative length [mm]

$\mathrm{L}_{\mathrm{c}} \quad$ cavitation length [mm]

$D_{\text {No }} \quad$ nozzle orifice diameter $[\mathrm{mm}]$

$D_{s} \quad$ sac diameter [mm]

$D_{\text {SC }} \quad$ normalized diameter of string cavitation [-]

$D_{v} \quad$ vortex diameter $[\mathrm{mm}]$

$\mathrm{P} \quad$ injection pressure [Mpa]

$\mathrm{P}_{\mathrm{b}} \quad$ back pressure [Mpa]

$\mathrm{P}_{\mathrm{v}} \quad$ vapour saturation pressure [Mpa]

$\mathrm{Re}_{1} \quad$ modified single Reynolds number [-]

$\mathrm{Re}_{\mathrm{s}} \quad$ modified twin Reynolds number [-]

$\mathrm{T}$ temperature $\left[{ }^{\circ} \mathrm{C}\right]$

$V_{\text {No }} \quad$ mean velocity in nozzle orifice $\left[\mathrm{m} \mathrm{s}^{-1}\right]$

$V_{\text {in }} \quad$ mean velocity at sac inlet $\left[\mathrm{m} \mathrm{s}^{-1}\right]$

$V_{s} \quad$ representative velocity $\left[\mathrm{m} \mathrm{s}^{-1}\right]$

$Z_{\text {in }} \quad$ needle lift [ $\mathrm{mm}$ ]

$Z_{\text {No }}$ distance between needle and orifice $[\mathrm{mm}]$ 


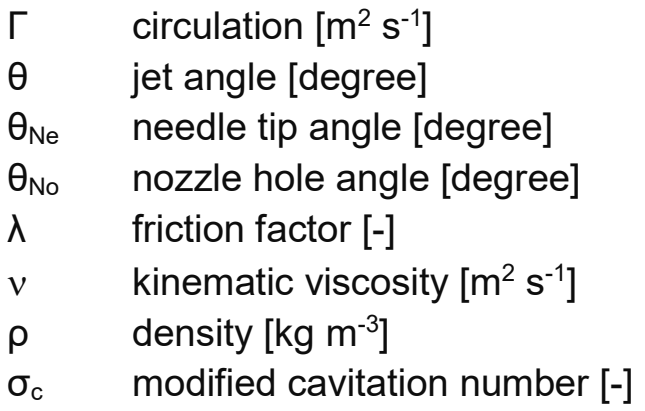

\section{References}

[1] Bergwerk, W., 1959, "Flow Pattern in Diesel Nozzle Spray Holes," Proc. Inst. Mech. Eng.

[2] Hayashi, T., Suzuki, M., and Ikemoto, M., 2013, "Effects of Internal Flow in a Diesel Nozzle on Spray Combustion," Int. J. Engine Res., 14(6), pp. 646-654.

[3] Hiroyasu, H., Arai, M., and Shimizu, M., 1991, "Break-up Length of a Liquid Jet and Internal Flow in a Nozzle," 13th Triennial International Conference on Liquid Atomization and Spray Systems, pp. 275-282.

[4] Sou, A., Hosokawa, S., and Tomiyama, A., 2007, "Effects of Cavitation in a Nozzle on Liquid Jet Atomization," Int. J. Heat Mass Transf., 50(17-18), pp. 3575-3582.

[5] Sou, A., Maulana, M. I., Isozaki, K., Hosokawa, S., and Tomiyama, A., 2008, "Effects of Nozzle Geometry on Cavitation in Nozzles of Pressure Atomizers," J. Fluid Sci. Technol., 3(5), pp. 622-632.

[6] Sou, A., Hosokawa, S., and Tomiyama, A., 2010, "Cavitation in Nozzles of Plain Orifice Atomizers with Various Length-to-Diameter Ratios," At. Sprays, 20(6), pp. 513-524.

[7] Theodorakakos, A., Mitroglou, N., and Gavaises, M., 2012, "Through Diesel Fuel Injectors," Proc. 8th Int. Symp. Cavitation, (September), pp. 1-7.

[8] Gavaises, M., Andriotis, A., Papoulias, D., Mitroglou, N., and Theodorakakos, A., 2009, "Characterization of String Cavitation in Large-Scale Diesel Nozzles with Tapered Holes," Phys. Fluids, 21(5).

[9] He, Z., Zhang, Z., Guo, G., Wang, Q., Leng, X., and Sun, S., 2016, "Visual Experiment of Transient Cavitating Flow Characteristics in the Real-Size Diesel Injector Nozzle," Int. Commun. Heat Mass Transf., 78, pp. 13-20.

[10] Pratama, R. H., Sou, A., Katsui, T., and Nishio, S., 2017, "String Cavitation in a Fuel Injector," At. Sprays, 27, pp. 189-205.

[11] Prasetya, R., Sou, A., Oki, J., Nakashima, A., Nishida, K., Wada, Y., Ueki, Y., and Yokohata, H., 2019, "Three-Dimensional Flow Structure and String Cavitation in a Fuel Injector and Their Effects on Discharged Liquid Jet," Int. J. Engine Res., pp. 1-14.

[12] Fitzgerald, R., Veccia, G., Peraza, J., and Martin, G., 2019, "Features of Internal Flow and Spray for a Multi-Hole Transparent Diesel Fuel Injector Tip," 29th European Conference on Liquid Atomization and Spray Systems - ILASS 2019, Paris.

[13] Nakamichi, K., Wakisaka, M., Kimura, K., Sou, A., Miwa, T., Nishida, K., Wada, Y., Ueki, Y., and Yokohata, H., 2020, "Four Dimensional Cavitation Flow in Multi-Hole Fuel Injector and Spray Behavior," Int. Conf. on Liquid Atomization and Sprays System (ICLASS)-Asia. 